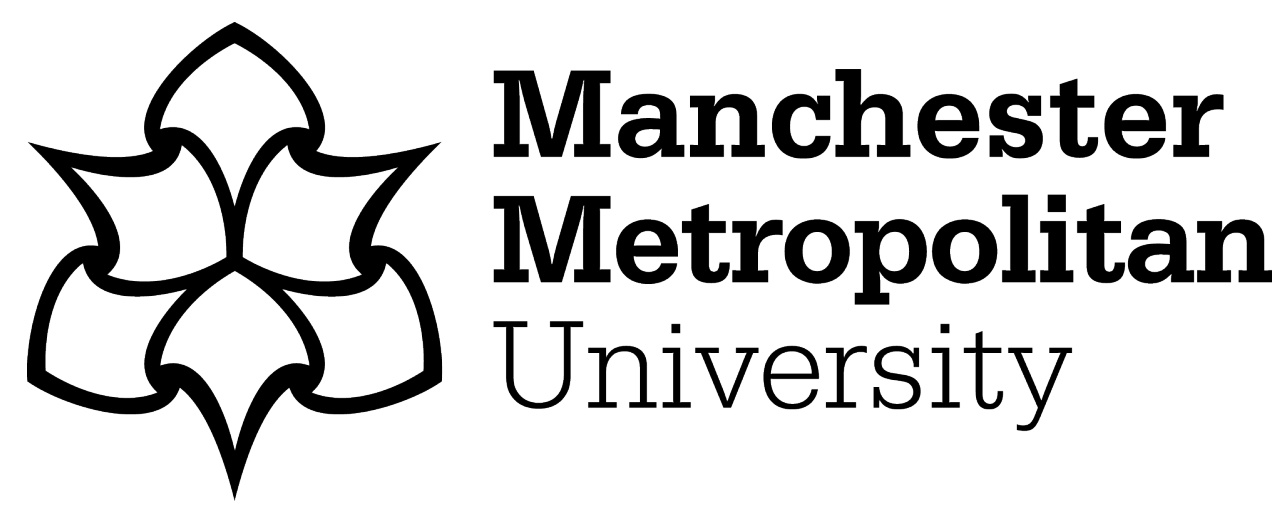

Ganse, Bergita, Bosutti, Alessandra, Drey, Michael and Degens, Hans ORCID logoORCID: https://orcid.org/0000-0001-7399-4841 (2021) Sixty days of head-down tilt bed rest with or without artificial gravity do not affect the neuromuscular secretome. Experimental Cell Research, 399 (2). p. 112463. ISSN 0014-4827

Downloaded from: https://e-space.mmu.ac.uk/627109/

Version: Published Version

Publisher: Elsevier

DOI: https://doi.org/10.1016/j.yexcr.2020.112463

Usage rights: Creative Commons: Attribution-Noncommercial-No Derivative Works 4.0

Please cite the published version 


\title{
Sixty days of head-down tilt bed rest with or without artificial gravity do not affect the neuromuscular secretome
}

\author{
Bergita Ganse $^{\mathrm{a},{ }^{*}}$, Alessandra Bosutti ${ }^{\mathrm{b}}$, Michael Drey ${ }^{\mathrm{c}}$, Hans Degens ${ }^{\mathrm{a}, \mathrm{d}}$ \\ ${ }^{a}$ Manchester Metropolitan University, Research Centre for Musculoskeletal Science \& Sports Medicine, Faculty of Science and Engineering, John Dalton Building, \\ Manchester, United Kingdom \\ ${ }^{\mathrm{b}}$ Department of Life Sciences, University of Trieste, Trieste, Italy \\ ${ }^{\mathrm{c}}$ Department of Medicine IV, Geriatrics, University Hospital of LMU Munich, Munich, Germany \\ ${ }^{\mathrm{d}}$ Lithuanian Sports University, Institute of Sport Science and Innovations, Kaunas, Lithuania
}

A R T I C L E I N F O

Keywords:

Astronaut

Microgravity

Disuse

Spaceflight

C-terminal agrin fragment

BDNF

\begin{abstract}
A B S T R A C T
Artificial gravity is a potential countermeasure to attenuate effects of weightlessness during long-term spaceflight, including losses of muscle mass and function, possibly to some extent attributable to disturbed neuromuscular interaction. The 60-day AGBRESA bed-rest study was conducted with 24 participants (16 men, 8 women; $33 \pm 9$ years; $175 \pm 9 \mathrm{~cm} ; 74 \pm 10 \mathrm{~kg} ; 8$ control group, 8 continuous (cAG) and 8 intermittent (iAG) centrifugation) to assess the impact of bed rest with or without daily 30-min continuous/intermittent centrifugation with $1 \mathrm{G}$ at the centre of mass. Fasting blood samples were collected before and on day 6, 20, 40 and 57 during $6^{\circ}$ head-down tilt bed rest. Concentrations of circulating markers of muscle wasting (GDF-8/myostatin; slow skeletal muscle troponin T; prostaglandin E2), neurotrophic factors (BDNF; GDNF) and C-terminal Agrin Fragment (CAF) were determined by ELISAs. Creatine kinase activity was assessed by colorimetric enzyme assay. Repeated-measures ANOVAs were conducted with TIME as within-subject, and INTERVENTION and SEX as between-subject factors. The analyses revealed no significant effect of bed rest or sex on any of the parameters. Continuous or intermittent artificial gravity is a safe intervention that does not have a negative impact of the neuromuscular secretome.
\end{abstract}

\section{Introduction}

Artificial gravity generated by human centrifugation is a potential countermeasure to mitigate the effects of weightlessness during longterm space missions [1-4]. Human centrifugation can be applied in a continuous or intermittent fashion. The efficacy of both alternatives has not yet been directly compared in a long-term bed-rest study. In a 5-day bed-rest study, intermittent centrifugation with $1 \mathrm{G}$ at the centre of mass was better tolerated than continuous centrifugation and was associated with lower adrenocortical stress responses [5] and attenuated reductions in orthostatic intolerance [6] compared to continuous centrifugation. In contrast to these positive effects of human centrifugation, neither continuous nor intermittent centrifugation counteracted bed-rest-induced changes in cardiac function [7] and the catabolic effects on muscle and bone metabolism [8]. However, in a 21-day bed-rest study, daily exposure to $1 \mathrm{~h}$ of continuous artificial gravity with $2.5 \mathrm{G}$ at the feet, similar to $1 \mathrm{G}$ at the centre of mass, had a small attenuating effect on losses in muscle mass and function, possibly mediated by a blunted increase in muscle myostatin content [9] and preserved rates of muscle protein synthesis [10]. It remains to be seen whether the attenuated bed-rest-induced atrophy after 21 days, but not after 5 days, is related to the duration and hence more accentuated after a long period of bed rest.

Deconditioning of the neuromuscular system, characterized by muscle weakness, is one of the major physiological responses to microgravity during long-term space missions and in bed rest [11-16, 63,65]. While muscle weakness during disuse is largely attributable to muscle atrophy, the contribution of an impaired motor drive may be an important factor for the loss of muscle strength, particularly during the early stages of disuse. Such impaired activation capacity may be the consequence of disuse-induced remodelling of the neuromuscular junctions (NMJ) [17] as an adaptation to the reduced number of nerve impulses reaching the motor end plate [18]. This adaptation of the NMJ can impair postsynaptic signal transduction and cause defects in the

\footnotetext{
* Corresponding author.

E-mail address: b.ganse@mmu.ac.uk (B. Ganse).
} 
excitation-contraction coupling [66]. The affected post-synaptic signalling can in turn activate apoptosis and proteolysis, and impair the regenerative potential of the muscle [19-21]. Neurotrophins and neurotrophic factors play an important role in maintaining the integrity of the NMJ $[18,22,23]$. Disuse-induced problems with neurotransmission and synaptic vesicle recycling may well be a consequence of a reduction in the levels of these circulating factors. It has been found that the circulating [24,25] and muscle [26-28] expression levels of some neurotrophic factors correlate positively with the extent of muscle loading. For example, in muscle the expression of glial cell line-derived neurotrophic factor (GDNF), important for maintenance of the NMJ [26,27], was markedly decreased with hind limb suspension and increased with exercise [28]. Daily treadmill exercise increased serum levels of GDNF and Brain Derived Neurotrophic Factor (BDNF) in rats undergoing peripheral nerve repair after nerve transection [25]. In line with these rodent studies, the bed-rest-induced increase in BDNF in old, but not young people, was suggested to be a neuroprotective response $[29,30]$. In addition to neuroprotection, the concomitant increase in circulating BDNF after muscle-damaging exercise and satellite cell activation [31] suggests that BDNF also facilitates muscle recovery from damage. Although no significant change in BDNF has been observed in young participants after 14 days of bed rest [29,30], it is possible that bed-rest-induced decrements in BDNF occur later in younger than older people and can be prevented by human centrifugation.

C-terminal Agrin Fragment (CAF) blood levels are suggested to be a biomarker for NMJ fragmentation and are used as a clinical marker for the progression of sarcopenia [32-34]. Elevated levels of the synaptic protease neurotrypsin cause a loss of NMJ integrity by fragmentation of agrin into the soluble $22 \mathrm{kDa}$ C-terminal Agrin Fragment that can lead to muscle wasting $[35,36]$. CAF levels have not been previously reported for bed rest or spaceflight, but are expected to rise in case of NMJ fragmentation during spaceflight and bed rest.

With regards to markers of muscle wasting, Creatine kinase and slow skeletal muscle troponin $\mathrm{T}$ are well-known makers and have been reported to be elevated in immobilization [37]. In addition, urine levels of prostaglandin E2 were decreased during Space Shuttle flights [38] and muscle myostatin levels were increased after 84 days of bed rest [39]. In a recent study on mice that spent 33 days on the International Space Station, it was shown that systemic inhibition of myostatin/activin A signalling led to increases in both muscle and bone mass, with comparable effects in ground and flight mice [40]. The myostatin signalling pathway is thus a potential target for the development of medications to serve as a countermeasure to muscle and bone loss. While the muscle levels of myostatin were elevated during bed rest, this was prevented by concomitant flywheel exercise [39], but nothing is known about the circulating levels of myostatin and the potential impact of human centrifugation.

To gain insight into the possible factors that affect the neuromuscular integrity during human centrifugation in a long-term bed-rest study we measured the time course of bed-rest-induced changes in neurotrophic factors, markers of muscle wasting and NMJ fragmentation. We hypothesized that bed rest is accompanied by transient changes in the levels of circulating neurotrophic factors, circulating markers of muscle wasting and C-terminal Agrin Fragment that are attenuated by intermittent and continuous artificial gravity.

\section{Methods}

\subsection{Ethical approval}

The study was approved by the IRB of North Rhine Medical Association (Ärztekammer Nordrhein, application number 2018143) in Düsseldorf, Germany, the IRB of Hospital Maastricht, The Netherlands (application number NL68345.068.18/METC 19-001), and by the IRB of NASA's Johnson Space Center, Houston, Texas, USA. It was registered in the German Clinical Trials Register (DRKS-ID: DRKS00015677).

\subsection{Experimental design}

The Artificial Gravity Bed Rest - European Space Agency (AGBRESA) study was a joint project of the European Space Agency (ESA) and the National Aeronautics and Space Administration (NASA). It was conducted at the German Aerospace Centre in Cologne, Germany, between March and December of 2019 in two campaigns with 12 participants each. The twenty-four healthy individuals ( 16 men, 8 women; $33 \pm 9$ years; $175 \pm 9 \mathrm{~cm} ; 74 \pm 10 \mathrm{~kg}$ ) spent three months in the 'Envihab' facility, with 15 days baseline data collection (BDC-15 to BDC1), 60 days of $6^{\circ}$ head-down tilt bed rest (HDBR, HDT1-HDT60) and 14 days of recovery ( $\mathrm{R}+0$ to $\mathrm{R}+13$ ) (Fig. 1 ). Sixteen ( 10 men, 6 women) of the participants were exposed to $30 \mathrm{~min}$ of daily human centrifugation and the other eight ( 6 men, 2 women) were in bed the entire time, serving as a control group. Participant characteristics are shown in Table 1.

Two modes of artificial gravity were compared: eight participants received human centrifugation in one continuous 30 -min bout $(n=8 ; 5$ men and 3 women), and the other eight were exposed to $6 \times 5$-min intermittent bouts with 3-min breaks in between bouts ( $\mathrm{n}=8$; 5 men and 3 women). Participants were pseudo-randomly assigned to groups for the first campaign, while for campaign 2 assignment aimed at demographic balancing of groups, as three women and one man dropped out and were subsequently replaced during the second campaign.

Each participant attended an information event, received a complete description of the experimental methods and study setup, and passed the medical and psychological testing. Medical screening for selection was similar to previous ESA HDBR studies [41]. In addition, participants had to pass a centrifuge tolerance test. All participants were informed about their right to withdraw from the study at any time.

\subsection{Head-down bed rest (HDBR)}

Bed rest was performed in $6^{0} \mathrm{HDBR}$ in accordance with the International Guidelines for Standardization of bed-rest studies in the spaceflight context [https://www.nasa.gov/sites/default/files/atoms/ files/bed_rest_studies_complete.pdf]. The day-night cycle included wake-up at 06:30 a.m. and lights-out at 22:00 p.m. During the HDT period, video-surveillance was applied to enforce strict bed rest, and all activities had to be conducted in the HDT position without a pillow (hygiene, toilet, reading, eating etc.). While remaining in $6^{\circ} \mathrm{HDT}$, participants were allowed to turn over, ensuring one shoulder was in contact with the bed at all times. During the ambulatory and rehabilitation periods, the participants were not authorized to leave the research facility or receive visitors. Leisure activities such as reading, playing games or using computers were permitted. Daytime sleeping or napping was not allowed.

\subsection{Application of short-arm centrifugation}

Continuous medical monitoring by physicians was conducted to ensure participant safety. The time-of-day for centrifugation was randomly assigned on a day-to-day basis. Participants were transferred to the centrifuge on a specific gurney to make sure the $-6^{\circ}$ HDT position was maintained during transport. They were asked to roll over to the centrifuge without using their leg muscles. During centrifugation, participants were exposed to $1 \mathrm{G}$ at their estimated centre of mass. The direction of centrifugation was alternated to ensure similar loading of both legs. Participants were instructed to stay calm, to not move their head and to keep their leg muscles relaxed. In case of low blood pressure, they were instructed to use the muscle pump in a way that was previously shown to them, without bending the knees. During a centrifuge run, the acceleration occurred at $5^{\circ} \cdot \mathrm{s}^{-2}$ for 32-33 s until the individual target rotation speed was reached, depending on the body size. Rotation at constant velocity then lasted either $30 \mathrm{~min}$ (continuous centrifugation) or $5 \times 6 \mathrm{~min}$, with 3 -min rest periods (intermittent centrifugation). 


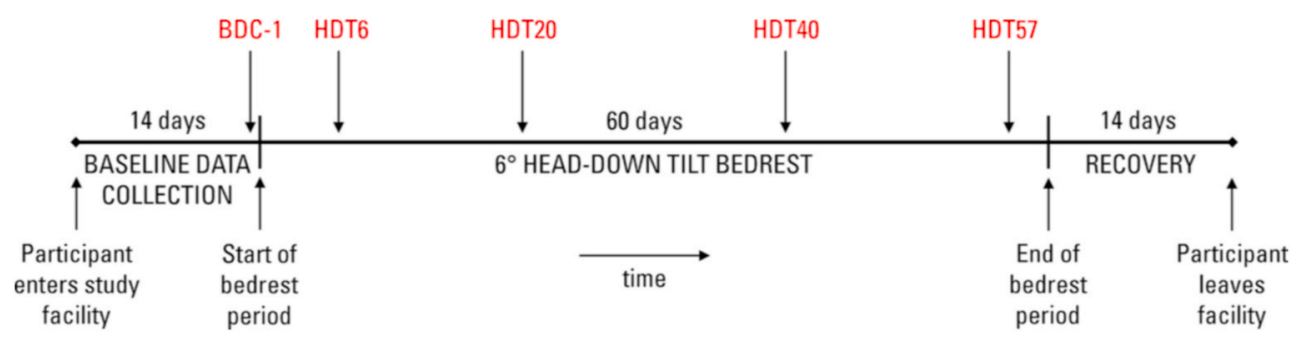

Fig. 1. Time schedule of each of the two campaigns. The time points of the blood draws for the present paper are highlighted in red.

Table 1

Participant characteristics at baseline; averages and standard deviations. ${ }^{\circ}$ One-way ANOVA; *Chi-square test.

\begin{tabular}{|c|c|c|c|c|}
\hline Variable & Control group & Continuous artificial gravity & Intermittent artificial gravity & Group difference $\mathrm{p}$ values \\
\hline$N$ & 8 & 8 & 8 & \\
\hline Age (years) & $32.3 \pm 7.4$ & $33.5 \pm 10.9$ & $34.0 \pm 10.1$ & $0.932^{\ddagger}$ \\
\hline Sex & 6 men, 2 women & 5 men, 3 women & 5 men, 3 women & $0.829^{*}$ \\
\hline Height $(\mathrm{cm})$ & $177 \pm 7$ & $172 \pm 8$ & $174 \pm 11$ & $0.598^{\mathrm{t}}$ \\
\hline Body mass $(\mathrm{kg})$ & $79.4 \pm 12.7$ & $71.8 \pm 10.2$ & $71.4 \pm 4.5$ & $0.203^{\mathrm{r}}$ \\
\hline Body mass index $\left(\mathrm{kg} / \mathrm{m}^{2}\right)$ & $25.2 \pm 2.6$ & $24.0 \pm 1.7$ & $23.6 \pm 1.6$ & $0.273^{\mathrm{r}}$ \\
\hline \multicolumn{5}{|l|}{ Markers of muscle wasting } \\
\hline Myostatin (ng/ml) & $3.19 \pm 1.03$ & $3.47 \pm 1.15$ & $3.28 \pm 0.75$ & $0.840^{\mathrm{f}}$ \\
\hline Slow Skeletal Muscle Troponin T (ng/ml) & $0.88 \pm 0.06$ & $0.95 \pm 0.11$ & $0.84 \pm 0.09$ & $0.071^{+}$ \\
\hline Creatine Kinase (mU/mL) & $19.8 \pm 10.8$ & $20.5 \pm 14.8$ & $25.7 \pm 9.3$ & $0.557^{\mathrm{t}}$ \\
\hline Prostaglandin E2 (ng/ml) & $1.43 \pm 0.22$ & $1.44 \pm 0.29$ & $1.34 \pm 0.21$ & $0.672^{\mathrm{f}}$ \\
\hline \multicolumn{5}{|l|}{ Neurotrophic factors } \\
\hline $\mathrm{BDNF}(\mathrm{ng} / \mathrm{ml})$ & $417 \pm 204$ & $573 \pm 246$ & $477 \pm 236$ & $0.360^{\mp}$ \\
\hline GDNF (ng/ml) & $69.3 \pm 69.9$ & $296.8 \pm 371.6$ & $87.8 \pm 121.2$ & $0.136^{t}$ \\
\hline \multicolumn{5}{|l|}{ NMJ integrity } \\
\hline C-terminal Agrin Fragment (pg/ml) & $129.2 \pm 209.3$ & $343.7 \pm 507.5$ & $64.5 \pm 26.7$ & $0.210^{+}$ \\
\hline
\end{tabular}

At the end of each run, deceleration was at $5^{\circ} \cdot \mathrm{s}^{-2}$ until the centrifuge had come to a complete stop.

\subsection{Analyses}

Fasting blood samples were collected on BDC-1, HDT6, HDT20, HDT40 and HDT57 always at the same time before breakfast. The blood was centrifugated and the aliquoted serum stored at $-80{ }^{\circ} \mathrm{C}$. The following serum parameters were determined in triplicates with the named commercially available detection kits.

1. GDF-8/Myostatin by the Quantikine ELISA of R\&D (DGDF80)

2. Slow skeletal muscle Troponin T by TnTs ELISA KIT of RayBiotech (ELH-TroponinT)

3. Creatine kinase activity by the colourimetric assay of Abcam (ab155901)

4. Prostaglandin E2 using the PGE2 high sensitivity ELISA Kit of Abcam (ab133055)

5. Brain derived neurotrophic factor by the ELISA Kit of RayBiotech (ELH-BDNF)

6. Glial cell line-derived neurotrophic factor by the DuoSet ELISA kit of R\&D (DY212)

7. C-terminal Agrin Fragment ELISA Kit of MyBioSource (MBS7606926)

All analyses were conducted according to the instructions by the manufacturer. The data from the plate reader were further processed using GraphPad Prism version 8.3.4 to compute serum concentrations or activity (creatine kinase) from the absorption values.

\subsection{Statistical analysis}

Normal distribution of data was tested by the Kolmogorov-Smirnov and Shapiro-Wilk tests. Due to non-normality, the data was transformed for troponin $\mathrm{T}(\log 10)$, BDNF $(1 / \mathrm{x})$, GDNF $(\log 10)$ and CAF $(1 / \mathrm{x})$ before conduction of further statistical analyses. To assess participant characteristics at baseline for differences in age, sex (chi-squared test), height, and weight between groups a one-way ANOVA was used. Repeated-measures ANOVA was used to determine the changes in Myostatin, Troponin T, Creatine kinase, PGE2, BDNF, GDNF and CAF at HDT6, HDT20, HDT40 and HDT57 compared to BDC-1, with TIME as a within-participant factor and INTERVENTION and SEX as betweensubject factors. AGE was added as a covariate. TIME*AGE, TIME*INTERVENTION and TIME*SEX interactions were also determined. Post-hoc tests used a Bonferroni correction to account for testing of multiple pairs. Significance was defined as $\mathrm{p}<0.05$.

\section{Results}

All blood samples were successfully obtained and processed as planned. At baseline, there was no significant difference between groups (Table 1). The analyses revealed no significant differences between men and women, and no significant effects of bed rest or the interventions on any of the measured parameters. In detail, the following findings were obtained.

\subsection{Circulating markers of muscle wasting}

Fig. 2 shows the time courses of the serum levels of GDF-8/myostatin (Fig. 2A), slow skeletal muscle troponin T (Fig. 2B), creatine kinase (Fig. 2C) and prostaglandin E2 (Fig. 2D). For GDF-8/myostatin, there was neither a time effect $(\mathrm{p}=0.935)$, nor a time*age $(\mathrm{p}=0.148)$, a time*intervention $(\mathrm{p}=0.878)$ or a time ${ }^{*}$ sex effect $(\mathrm{p}=0.306)$. The same was true for the slow skeletal muscle troponin $\mathrm{T}$ (time, $\mathrm{p}=0.543$; time*age, $\mathrm{p}=0.375$; time*intervention, $\mathrm{p}=0.183$; time*sex, $\mathrm{p}=$ $0.159)$. No time effects or interactions were found for creatine kinase (time, $\mathrm{p}=0.638$; time*age, $\mathrm{p}=0.821$; time*intervention, $\mathrm{p}=0.373$; time*sex, $\mathrm{p}=0.058$ ) and prostaglandin $\mathrm{E} 2$ either (time, $\mathrm{p}=0.339$; time*age, $\mathrm{p}=0.678$; time*intervention, $\mathrm{p}=0.250$; time*sex, $\mathrm{p}=$ 0.391). 


\subsection{Neurotrophic factors}

Fig. 3 shows the time courses of the serum BDNF (Fig. 3A) and GDNF (Fig. 3B) levels. For both parameters, there was neither a time effect (BDNF, $\mathrm{p}=0.650$; GDNF $\mathrm{p}=0.523$ ), nor a time*age (BDNF $\mathrm{p}=0.382$; GDNF $\mathrm{p}=0.436)$, time*intervention (BDNF $\mathrm{p}=0.340$; GDNF $\mathrm{p}=0.820$ ) or time*sex (BDNF $\mathrm{p}=0.378$; GDNF $\mathrm{p}=0.996$ ) interaction.

\subsection{C-terminal agrin fragment}

Fig. 4 depicts changes in the serum concentration of the C-terminal Agrin Fragment. No time-effect $(p=0.549)$, time*age $(p=0.748)$, time*intervention $(0.479)$ or time*sex $(\mathrm{p}=0.511)$ interaction was found.

\section{Discussion}

The aim of the present study was to assess 1) the immobilization effects of bed rest on the neuromuscular interaction secretome and 2) whether any immobilization-induced changes in the secretome could be attenuated by daily continuous or intermittent human centrifugation with $1 \mathrm{G}$ at the centre of mass. The main finding was that none of the measured parameters including serum markers of muscle wasting (GDF8/myostatin, slow skeletal muscle troponin $\mathrm{T}$, creatine kinase, prostaglandin E2), neurotrophic factors (BDNF, GDNF) and C-terminal Agrin Fragment, was affected by bed rest or human centrifugation.

\subsection{Study design}

The AGBRESA study was the first long-term bed-rest study to compare continuous vs. intermittent human centrifugation as a countermeasure. The 24 participants of both sexes were divided over three groups (control, continuous and intermittent artificial gravity). The centrifugation was of a similar, if anything slightly lower, G-intensity and shorter duration than previous studies (30 vs. $60 \mathrm{~min}$ ) $[9,10]$. Artificial gravity was applied without an additional exercise
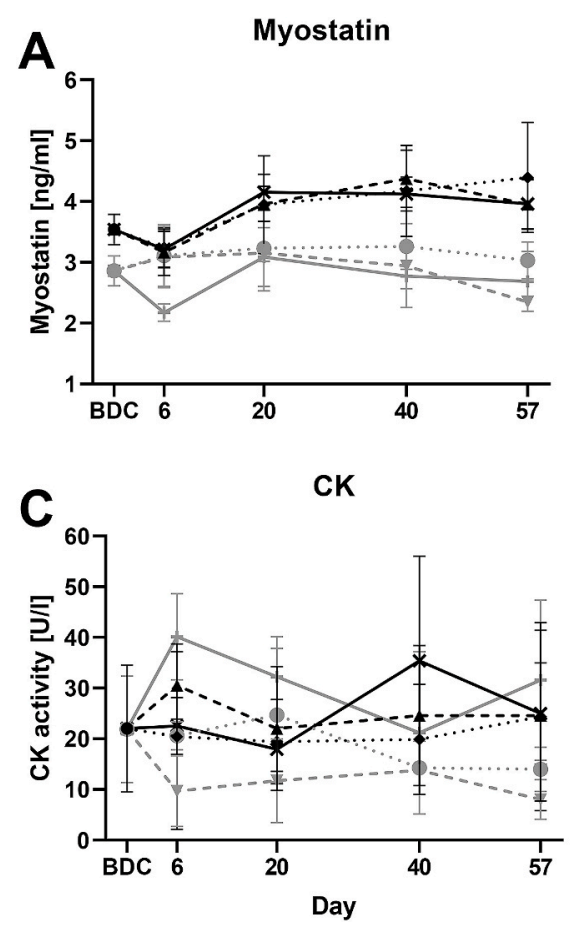

countermeasure, so data may serve as a control for future bed-rest studies that will test different types of exercise interventions during centrifugation. For instance, supine cycling that attenuated the reduction in aerobic capacity and orthostatic tolerance in bed rest [42] and resistive exercise that is applied on the International Space Station [43] are potential candidates to be implemented on human centrifuges. The set of analyses we conducted in the AGBRESA study has not been previously reported in the context of human centrifugation.

\subsection{Circulating markers of muscle wasting}

GDF-8/Myostatin is a TGF- $\beta$ superfamily protein produced by muscle cells that inhibits muscle growth and myoblast proliferation, and thereby is a negative regulator of muscle mass [44]. It is a potential target for new treatment options for sarcopenia and frailty $[40,45,46]$. Indeed, in mice systemic inhibition of myostatin/activin A signalling during spaceflight led to increases in both muscle and bone mass [40]. This may also be significant in humans as Irimia at al [39] reported an increased muscle myostatin content after 84 days of bed rest, and in a 21-days bed-rest study, the increases in muscle myostatin content were blunted by $60 \mathrm{~min}$ of daily centrifugation [9]. The results of the present study neither showed increased serum levels with bed rest nor a blunting effect of continuous or intermittent centrifugation. Part of the discrepancy between these previous and our studies may be related to the fact that we measured circulating myostatin, whereas in the other studies the muscle levels of myostatin were determined [9,39]. Future studies may reveal the relationship between muscle and circulating levels of myostatin, but our study indicates that circulating myostatin is not altered during bed rest or centrifugation.

Binding of $\mathrm{Ca}^{2+}$ to troponin results in the conformational change of tropomyosin that exposes the myosin binding sites on actin thereby enabling cross-bridge formation and muscle contraction [47]. This protein is an integral part of the muscle filaments and muscle breakdown may cause the release of troponin into the blood stream, as observed in myocardial infarction [48]. Likewise, creatine kinase, an enzyme that generates adenosine triphosphate (ATP) from phosphocreatine and

\section{B Troponin T}
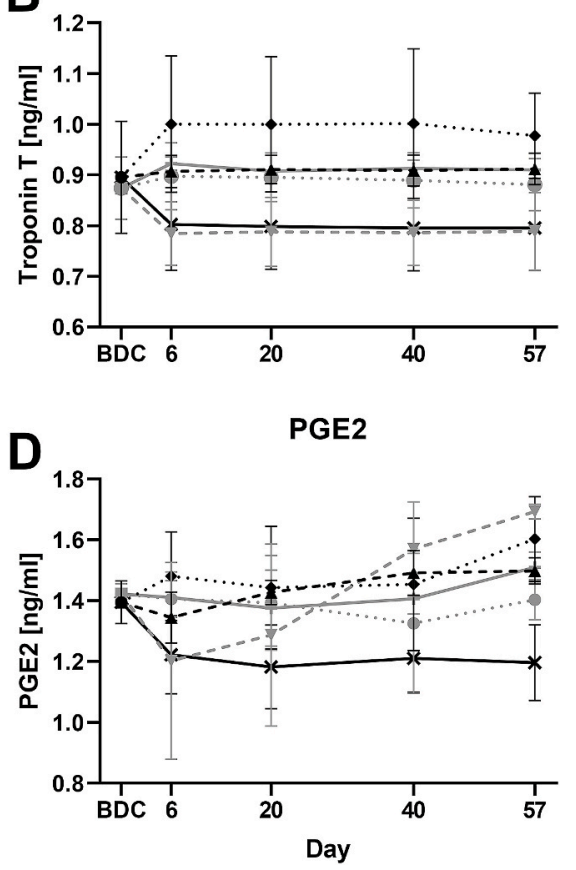

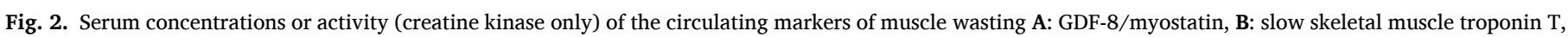

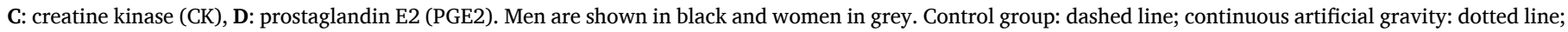
intermittent artificial gravity: continuous line. Data are shown as mean \pm SEM. 

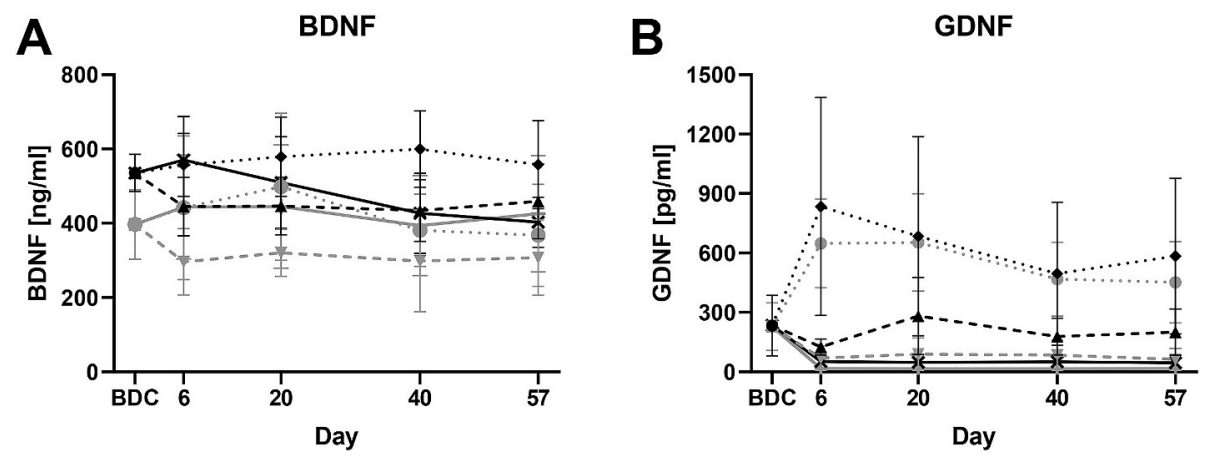

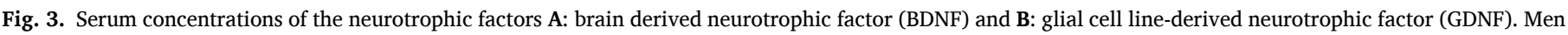

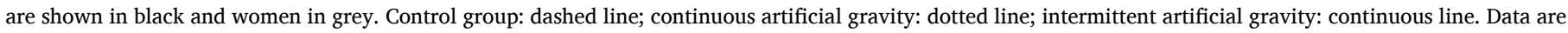
shown as mean \pm SEM.

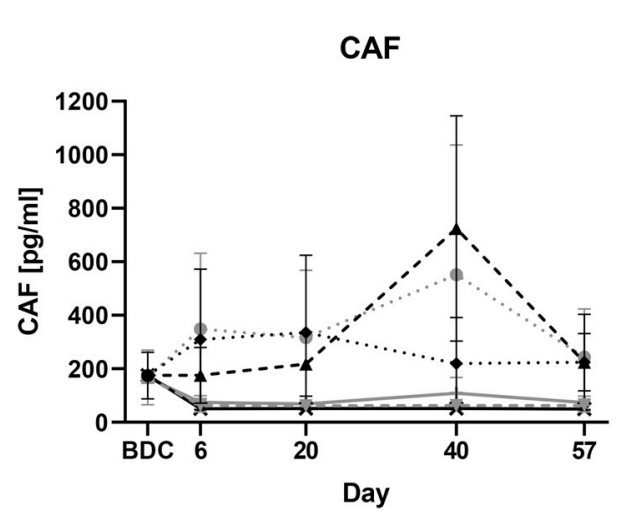

Fig. 4. Serum concentrations of C-terminal Agrin Fragment. Men are shown in black and women in grey. Control group: dashed line; continuous artificial gravity: dotted line; intermittent artificial gravity: continuous line. Data are shown as mean \pm SEM.

adenosine diphosphate required for muscle contraction [49] is released in the blood stream during muscle breakdown [48]. In the present study, we did not find the increased serum levels of muscle slow troponin or creatine kinase that would be expected in case of muscle damage or degradation. This thus suggests that the bed-rest-induced atrophy is not associated with damage, but is a controlled process of muscle protein breakdown.

Prostaglandin E2 has many functions in the body, including to cause fever, inflammation, oedema and uterus contractions. In the nervous system, prostaglandin E2 may also lead to inflammation [50]. It is also involved in the muscle-bone crosstalk [51,52]. In parabolic flights and 4 days of spaceflight, increased PGE2 levels were found in osteoblastic cell medium [53,54], while Stein et al. [38] reported decreased urine levels of prostaglandin E2 during Space Shuttle flights. Here we found no change in circulating prostaglandin E2 levels during bed rest. It is thus equivocal whether muscle unloading and decreased physical activity cause a rise or decrease in PGE2, but part of the discrepancy may well be related to the different systems investigated, ranging from cells (increase) to the whole body (space shuttle and our present study). Another possible explanation is that real microgravity activates cell pathways and stress responses in different ways than bed rest, where the gravitational pull is still present.

\subsection{Neurotrophic factors}

BDNF is a nerve growth factor that stimulates survival and growth of neurons of the central and peripheral nervous system [64]. It can be secreted by the central nervous system, but also other tissues, such as liver, heart, lung [55] and skeletal muscle [56]. Increases in BDNF were found in the older ( $60 \pm 4$ years) but not in the younger ( $23 \pm 3$ years) participants of a 14-day bed-rest study in Slovenia [29,30]. The average age in the present bed-rest study was $33.3 \pm 9.2$ years, so the absence of an increase in BDNF serum levels was in line with those previous findings in younger participants. Additional artificial gravity to bed rest did not result in any change in BDNF serum concentrations. This corresponds with the observation that wheelchair rugby training did not induce an increase in BDNF in otherwise sedentary chronic cervical spinal cord injury participants [57] and suggests that exercise does not have an effect on BDNF serum levels during periods of disuse.

GDNF is a protein expressed in glial cells of the brain and can be secreted by skeletal muscle [58]. It plays a role in neuron survival and maintenance of the NMJ $[26,27,58]$. It has been shown in rats that the daily treadmill exercise-induced improved peripheral nerve regeneration correlated with an increase in both GDNF and BDNF expression [25]. While exercise may increase GDNF expression, little is known about the impact of disuse. Kulikova et al. [59] studied the expression of genes related to brain neuroplasticity, including GDNF, in mice after one month of hindlimb-suspension compared to mice that had spent one month on board Russian biosatellite Bion-M1. No changes in GDNF genes were found in the hindlimb-suspension group while the spaceflight group had decreased expressions of GDNF genes in the striatum and substantia nigra. The ground-based model also failed to show the changes in the serotonin and dopaminergic systems, as well as some apoptotic factors that were observed in spaceflight. This is in line with the present study, as we observed no significant changes in GDNF serum concentrations. Perhaps disuse per se, other than real microgravity, has no effects on GDNF.

\subsection{C-terminal agrin fragment}

Agrin is a basal lamina protein that promotes the clustering of nAChRs and other proteins at the NMJ [60]. Elevated levels of the synaptic protease neurotrypsin, as seen during ageing, cause loss of integrity of the NMJ by degradation of agrin into the soluble $22 \mathrm{kDa}$ C-terminal agrin fragment that can ultimately lead to muscle wasting $[35,36]$. The presence of C-terminal agrin fragment (CAF) in human serum thus represents a potential biomarker of NMJ fragmentation. While elevated levels of C-terminal agrin fragment have been found in patients with sarcopenia [32-34], exposure to bed rest with or without human centrifugation did not lead to increases in serum CAF concentrations in the present study.

The absence of changes in serum markers of muscle wasting, neurotrophic factors and C-terminal agrin fragment corresponds with the absence of changes in the number (MUNIX) and size (MUSIX) of motor units found in the same bed-rest study [61] and normal muscle activation during a fatigue test after 21 days bed rest [13]. In combination, these observations suggest that the neuromuscular interaction is not compromised during bed rest of up to 60 days. It is possible, however, 
that longer durations of bed rest will have a negative impact on the neuromuscular integrity, as there are morphological indications of remodelling of the NMJ during bed rest [62].

\subsection{Limitations}

The main limitation of the present study was a high variation in a relatively small, but for bed-rest studies common sample size. Although there were only 8 women (vs 16 men) in the study, the absence of a sex effect and sex interactions indicates that this was not a major issue.

\section{Conclusions}

Our findings indicate that long-term bed rest or artificial gravity with $1 \mathrm{G}$ at the centre of mass for $30 \mathrm{~min}$ per day had no effects on the neuromuscular secretome. Artificial gravity neither resulted in changes in neurotrophic factors such as BDNF, GDNF or CAF, nor in circulating markers of muscle wasting, indicating that it is a safe intervention that does not have negative effects on neuromuscular integrity.

\section{Data availability statement}

The data that support the findings of this study are available from the corresponding author upon reasonable request.

\section{Role of the funding source}

The authors acknowledge the German Research Foundation (DFG, grant number GA 2420/1-1), German Aerospace Center (DLR, grant number 50WB1928) and the UK Space Agency (ST/S0001735/1 \& ST/ T00066X/1) in funding this project. In addition, the authors appreciate the support of the European Space Agency (ESA, 16-16ESA AGBR-0013, contract number 4000113871/15/NL/PG) and the National Aeronautics and Space Administration (NASA, contract number 80JSC018P0078). The DFG provided a research fellowship grant to BG. The UK Space Agency and DLR paid for the consumables. ESA and NASA were involved in the study design of the overall bedrest study but not in the experiments we conducted on the blood samples. The funders were not involved in the collection, analysis and interpretation of data; in the writing of the report; and in the decision to submit the article for publication.

\section{Credit author statement}

Bergita Ganse, Conceptualization, Investigation, Data curation, Visualization, Writing - original draft preparation. Alessandra Bosutti, Methodology, Writing-reviewing and editing. Michael Drey, Funding acquisition, Supervision, Writing-reviewing and editing. Hans Degens, Resources, Supervision, Formal analysis, Writing-reviewing and editing.

\section{Declaration of competing interest}

The authors declare that they have no known competing financial interests or personal relationships that could have appeared to influence the work reported in this paper.

\section{Acknowledgements}

Special thanks for the great operational support of Dr. Jennifer NgoAnh (ESA), Dr. Edwin Mulder, Dr. Jessica Lee and the entire team of the DLR Institute of Aerospace Medicine in Cologne during the study.

\section{References}

[1] R.R. Burton, L.J. Meeker, Physiologic validation of a short-arm centrifuge for space application, Aviat Space Environ. Med. 63 (6) (1992) 476-481.
[2] G. Clément, A. Pavy-Le Traon, Centrifugation as a countermeasure during actual and simulated microgravity: a review, Eur. J. Appl. Physiol. 92 (3) (2004) $235-248$.

[3] S.M. Smith, S.R. Zwart, M.A. Heer, N. Baecker, H.J. Evans, A.H. Feiveson, L. C. Shackelford, A.D. Leblanc, Effects of artificial gravity during bed rest on bone metabolism in humans, J. Appl. Physiol. 107 (1) (2009) 47-53.

[4] L.R. Young, W.H. Paloski, Short radius intermittent centrifugation as a countermeasure to bed-rest and 0-G deconditioning: IMAG pilot study summary and recommendations for research, J. Gravitational Physiol. 14 (1) (2007) P31-P33.

[5] A. Choukèr, B. Feuerecker, S. Matzel, I. Kaufmann, C. Strewe, M. Hoerl, G. Schelling, M. Feuerecker, Psychoneuroendocrine alterations during 5 days of head-down tilt bed rest and artificial gravity interventions, Eur. J. Appl. Physiol. 113 (8) (2013) 2057-2065.

[6] D. Linnarsson, R.L. Hughson, K.S. Fraser, G. Clément, L.L. Karlsson, E. Mulder, W. H. Paloski, J. Rittweger, F.L. Wuyts, J. Zange, Effects of an artificial gravity countermeasure on orthostatic tolerance, blood volumes and aerobic power after short-term bed rest (BR-AG1), J. Appl. Physiol. 118 (1) (2015) 29-35.

[7] E.G. Caiani, P. Massabuau, L. Weinert, P. Vaïda, R.M. Lang, Effects of 5 days of head-down bed rest, with and without short-arm centrifugation as countermeasure, on cardiac function in males (BR-AG1 study), J. Appl. Physiol. 117 (6) (2014) 624-632.

[8] J. Rittweger, M.P. Bareille, G. Clément, D. Linnarsson, W.H. Paloski, F. Wuyts, J. Zange, O. Angerer, Short-arm centrifugation as a partially effective musculoskeletal countermeasure during 5-day head-down tilt bed rest-results from the BRAG1 study, Eur. J. Appl. Physiol. 115 (6) (2015) 1233-1244.

[9] V.J. Caiozzo, F. Haddad, S. Lee, M. Baker, W.H. Paloski, K.M. Baldwin, Is artificial gravity an effective countermeasure to microgravity: effects on knee extensor and plantar flexor muscle groups, J. Appl. Physiol. 107 (1) (2009) 39-46.

[10] T.B. Symons, M. Sheffield-Moore, D.L. Chinkes, A.A. Ferrando, D. Paddon-Jones, Artificial gravity maintains skeletal muscle protein synthesis during 21 days of simulated microgravity, J. Appl. Physiol. 107 (1) (2009) 34-38.

[11] A. Bosutti, G. Malaponte, M. Zanetti, P. Castellino, M. Heer, G. Guarnieri, G. Biolo, Calorie restriction modulates inactivity-induced changes in the inflammatory markers C-reactive protein and pentraxin-3, J. Clin. Endocrinol. Metab. 93 (8) (2008) 3226-3229.

[12] A. Bosutti, M. Salanova, D. Blottner, J. Buehlmeier, E. Mulder, J. Rittweger, M. H. Yap, B. Ganse, H. Degens, Whey protein with potassium bicarbonate supplement attenuates the reduction in muscle oxidative capacity during 19 days bed rest, J. Appl. Physiol. 121 (4) (2016) 838-848.

[13] A. Bosutti, E. Mulder, J. Zange, J. Bühlmeier, B. Ganse, H. Degens, Effects of 21 days of bed rest and whey protein supplementation on plantar flexor muscle fatigue resistance during repeated shortening contractions, Eur. J. Appl. Physiol. 120 (2020) 969-983.

[14] T. Debevec, B. Ganse, U. Mittag, O. Eiken, I.B. Mekjavic, J. Rittweger, Hypoxia aggravates inactivity-related muscle wasting, Front. Physiol. 9 (2018) 494.

[15] H. Degens, S.E. Alway, Control of muscle size during disuse, disease, and aging, Int. J. Sports Med. 27 (2006) 94-99.

[16] M.V. Narici, M.D. de Boer, Disuse of the musculo-skeletal system in space and on earth, Eur. J. Appl. Physiol. 113 (2011) 403-420.

[17] R.R. Roy, K.M. Baldwin, V.R. Edgerton, Response of the neuromuscular unit to spaceflight: what has been learned from the rat model, Exerc. Sport Sci. Rev. 24 (1996) 399-425.

[18] M.R. Deschenes, Motor unit and neuromuscular junction remodeling with aging, Curr. Aging Sci. 4 (2011) 209-220.

[19] M. Crompton, The mitochondrial permeability transition pore and its role in cell death, Biochem. J. 341 (1999) 233-249.

[20] A.M. Mukhina, E.G. Altaeva, T.L. Nemirovskaya, B.S. Shenkman, The role of L-type calcium channels in the accumulation of $\mathrm{Ca} 2+$ in soleus muscle fibers in the rat and changes in the ratio of myosin and SERCA isoforms in conditions of gravitational unloading, Neurosci. Behav. Physiol. 92 (2008) 1285-1295.

[21] M. Salanova, G. Schiffl, D. Blottner, Atypical fast SERCA1a protein expression in slow myofibers and differential S-nitrosylation prevented by exercise during long term bed rest, Histochem. Cell Biol. 132 (2009) 383-394.

[22] A.M. Corse, M.M. Bilak, S.R. Bilak, M. Lehar, J.D. Rothstein, R.W. Kuncl, Preclinical testing of neuroprotective neurotrophic factors in a model of chronic motor neuron degeneration, Neurobiol. Dis. 6 (1999) 335-346.

[23] K. Sakuma, A. Yamaguchi, The recent understanding of the neurotrophin's role in skeletal muscle adaptation, J. Biomed. Biotechnol. 2011 (2011) 201696.

[24] L.T. Ferris, J.S. Williams, C.L. Shen, The effect of acute exercise on serum brainderived neurotrophic factor levels and cognitive function, Med. Sci. Sports Exerc. 39 (2007) 728-734.

[25] J.S. Park, A. Höke, Treadmill exercise induced functional recovery after peripheral nerve repair is associated with increased levels of neurotrophic factors, PloS One 9 (2014), e90245.

[26] Q.T. Nguyen, A.S. Parsadanian, W.D. Snider, J.W. Lichtman, Hyperinnervation of neuromuscular junctions caused by GDNF overexpression in muscle, Science 279 (5357) (1998) 1725-1729.

[27] M. Zwick, L. Teng, X. Mu, J.E. Springer, B.M. Davis, Overexpression of GDNF induces and maintains hyperinnervation of muscle fibers and multiple endplate formation, Exp. Neurol. 171 (2001) 342-350.

[28] E.A. Wehrwein, E.M. Roskelley, J.M. Spitsbergen, GDNF is regulated in an activitydependent manner in rat skeletal muscle, Muscle Nerve 26 (2002) 206-211.

[29] A. Passaro, C. Soavi, U. Marusic, E. Rejc, J.M. Sanz, M.L. Morieri, E.D. Nora, V. Kavcic, M.V. Narici, C. Reggiani, G. Biolo, G. Zuliani, S. Lazzer, R. Pišot, Computerized cognitive training and brain derived neurotrophic factor during bed 
rest: mechanisms to protect individual during acute stress, Aging (Albany NY) 9 (2) (2017) 393-407.

[30] C. Soavi, U. Marušič, J.M. Sanz, M.L. Morieri, E. Dalla Nora, B. Šimunič, R. Pišot, G. Zuliani, A. Passaro, Age-related differences in plasma BDNF levels after prolonged bed rest, J. Appl. Physiol. 120 (10) (2016) 1118-1123.

[31] B.R. McKay, J.P. Nederveen, S.A. Fortino, T. Snijders, S. Joanisse, D.A. Kumbhare, G. Parise, Brain-derived neurotrophic factor is associated with human muscle satellite cell differentiation in response to muscle-damaging exercise, Appl. Physiol. Nutr. Metabol. 45 (6) (2020) 581-590.

[32] R. Qaisar, A. Karim, T. Muhammad, Plasma CAF22 levels as a useful predictor of muscle health in patients with chronic obstructive pulmonary disease, Biology 9 (7) (2020) 166.

[33] C. Sánchez-Castellano, S. Martín-Aragón, P. Bermejo-Bescós, N. Vaquero-Pinto, C. Miret-Corchado, A. Merello de Miguel, A.J. Cruz-Jentoft, Biomarkers of sarcopenia in very old patients with hip fracture, J Cachexia Sarcopenia Muscle 11 (2) (2020) 478-486.

[34] N. Scherbakov, M. Knops, N. Ebner, M. Valentova, A. Sandek, U. Grittner, P. Dahinden, S. Hettwer, J.C. Schefold, S. von Haehling, S.D. Anker, M. Joebges, W. Doehner, Evaluation of C-terminal Agrin Fragment as a marker of muscle wasting in patients after acute stroke during early rehabilitation, J Cachexia Sarcopenia Muscle 7 (1) (2016) 60-67.

[35] M.F. Bolliger, A. Zurlinden, D. Lüscher, L. Bütikofer, O. Shakhova, M. Francolini, S. V. Kozlov, P. Cinelli, A. Stephan, A.D. Kistler, T. Rülicke, P. Pelczar, B. Ledermann, G. Fumagalli, S.M. Gloor, B. Kunz, P. Sonderegger, Specific proteolytic cleavage of agrin regulates maturation of the neuromuscular junction, J. Cell Sci. 123 (Pt 22) (2010) 3944-3955.

[36] M. Drey, C.C. Sieber, J.M. Bauer, W. Uter, P. Dahinden, R.G. Fariello, J. W. Vrijbloed, FiAT intervention group, C-terminal Agrin Fragment as a potential marker for sarcopenia caused by degeneration of the neuromuscular junction, Exp. Gerontol. 48 (2013) 76-80.

[37] J. St-Amand, K. Okamura, K. Matsumoto, S. Shimizu, Y. Sogawa, Characterization of control and immobilized skeletal muscle: an overview from genetic engineering, FASEB J 15 (3) (2001) 684-692.

[38] T.P. Stein, M.D. Schluter, L.L. Moldawer, Endocrine relationships during human spaceflight, Am. J. Physiol. 276 (1 Pt 1) (1999) E155-E162.

[39] J.M. Irimia, M. Guerrero, P. Rodriguez-Miguelez, J.A. Cadefau, P.A. Tesch, R. Cussó, R. Fernandez-Gonzalo, Metabolic adaptations in skeletal muscle after 84 Days of bed rest with and without concurrent flywheel resistance exercise, J. Appl. Physiol. 122 (1) (2017) 96-103.

[40] S.J. Lee, A. Lehar, Meir Ju, C. Koch, A. Morgan, L.E. Warren, R. Rydzik, D. W. Youngstrom, H. Chandok, J. George, J. Gogain, M. Michaud, T.A. Stoklasek, Y. Liu, E.L. Germain-Lee, Targeting myostatin/activin A protects against skeletal muscle and bone loss during spaceflight, Proc. Natl. Acad. Sci. U. S. A. 117 (38) (2020) 23942-23951.

[41] J.S. Lawley, L.G. Petersen, E.J. Howden, S. Sarma, W.K. Cornwell, R. Zhang, L. A. Whitworth, M.A. Williams, B.D. Levine, Effect of gravity and microgravity on intracranial pressure, J. Physiol. 595 (6) (2017) 2115-2127.

[42] S. Shibata, M. Perhonen, B.D. Levine, Supine cycling plus volume loading prevent cardiovascular deconditioning during bed rest, J. Appl. Physiol. 108 (2010) 1177-1186.

[43] S.M. Smith, M.A. Heer, L.C. Shackelford, J.D. Sibonga, L. Ploutz-Snyder, S. R. Zwart, Benefits for bone from resistance exercise and nutrition in longduration spaceflight: evidence from biochemistry and densitometry, J. Bone Miner. Res. 27 (2012) 1896-1906.

[44] S.J. Lee, Extracellular regulation of myostatin: a molecular rheostat for muscle mass, Immunol. Endocr. Metab. Agents Med. Chem. 10 (2010) 183-194.

[45] J. Chew, L. Tay, J.P. Lim, B.P. Leung, A. Yeo, S. Yew, Y.Y. Ding, W.S. Lim, Serum myostatin and IGF-1 as gender-specific biomarkers of frailty and low muscle mass in community-dwelling older adults, J. Nutr. Health Aging 23 (10) (2019) 979-986.

[46] A. de Sire, A. Baricich, F. Renò, C. Cisari, N. Fusco, M. Invernizzi, Myostatin as a potential biomarker to monitor sarcopenia in hip fracture patients undergoing a multidisciplinary rehabilitation and nutritional treatment: a preliminary study, Aging Clin. Exp. Res. 32 (5) (2020) 959-962.
[47] A. Mondal, J.P. Jin, Protein structure-function relationship at work: learning from myopathy mutations of the slow skeletal muscle isoform of troponin T, Front. Physiol. 7 (2016) 449.

[48] S.G. Williams, J.H. Silas, F. Joseph, M. Jackson, D. Rittoo, T. Currie, Troponin T: how high is high? Relationship and differences between serum cardiac markers according to level of creatine kinase and type of myocardial infarction, Postgrad. Med. 80 (948) (2004) 613-614.

[49] C.J. Barclay, Energy demand and supply in human skeletal muscle, J. Muscle Res. Cell Motil. 38 (2) (2017) 143-155.

[50] K. Tsuge, T. Inazumi, A. Shimamoto, Y. Sugimoto, Molecular mechanisms underlying prostaglandin E2-exacerbated inflammation and immune diseases, Int. Immunol. 31 (9) (2019) 597-606.

[51] G. Li, L. Zhang, D. Wang, L. AIQudsy, J.X. Jiang, H. Xu, P. Shang, Muscle-bone crosstalk and potential therapies for sarco-osteoporosis, J. Cell. Biochem. 120 (9) (2019) 14262-14273.

[52] C. Tagliaferri, Y. Wittrant, M.J. Davicco, S. Walrand, V. Coxam, Muscle and bone, two interconnected tissues, Ageing Res. Rev. 21 (2015) 55-70.

[53] A. Guignandon, L. Vico, C. Alexandre, M.H. Lafage-Proust, Shape changes of osteoblastic cells under gravitational variations during parabolic flightrelationship with PGE2 synthesis, Cell Struct. Funct. 20 (1995) 369-375.

[54] M. Hughes-Fulford, M.L. Lewis, Effects of microgravity on osteoblast growth activation, Exp. Cell Res. 224 (1996) 103-109.

[55] M. Miranda, J.F. Morici, M.B. Zanoni, P. Bekinschtein, Brain-derived neurotrophic factor: a key molecule for memory in the healthy and the pathological brain, Front. Cell. Neurosci. 13 (2019) 363.

[56] V.B. Matthews, M.B. Aström, M.H. Chan, C.R. Bruce, K.S. Krabbe, O. Prelovsek, T. Akerström, C. Yfanti, C. Broholm, O.H. Mortensen, M. Penkowa, P. Hojman, A. Zankari, M.J. Watt, H. Bruunsgaard, B.K. Pedersen, M.A. Febbraio, Brainderived neurotrophic factor is produced by skeletal muscle cells in response to contraction and enhances fat oxidation via activation of AMP-activated protein kinase, Diabetologia 52 (7) (2009) 1409-1418.

[57] E. Sadowska-Krępa, A. Zwierzchowska, M. Głowacz, K. Borowiec-Rybak, B. Kłapcińska, Blood metabolic response to a long-term wheelchair rugby training, Spinal Cord 54 (5) (2016) 371-375.

[58] A.F. Cintrón-Colón, G. Almeida-Alves, A.M. Boynton, J.M. Spitsbergen, GDNF synthesis, signaling, and retrograde transport in motor neurons, Cell Tissue Res. 382 (1) (2020) 47-56.

[59] E.A. Kulikova, V.A. Kulikov, N.A. Sinyakova, A.V. Kulikov, N.K. Popova, The effect of long-term hindlimb unloading on the expression of risk neurogenes encoding elements of serotonin-, dopaminergic systems and apoptosis; comparison with the effect of actual spaceflight on mouse brain, Neurosci. Lett. 640 (2017) 88-92.

[60] F. Rupp, D.G. Payan, C. Magill-Solc, D.M. Cowan, R.H. Scheller, Structure and expression of a rat agrin, Neuron 6 (5) (1991) 811-823.

[61] J. Attias, A. Grassi, A. Bosutti, B. Ganse, H. Degens, M. Drey, Head-down tilt bed rest with or without artificial gravity is not associated with motor unit remodeling, Eur. J. Appl. Physiol. 120 (2020) 2407-2415.

[62] M. Salanova, E. Bortoloso, G. Schiffl, M. Gutsmann, D.L. Belavy, D. Felsenberg, S. Furlan, P. Volpe, D. Blottner, Expression and regulation of Homer in human skeletal muscle during neuromuscular junction adaptation to disuse and exercise, FASEB J 25 (12) (2011) 4312-4325.

[63] G.R. Adams, V.J. Caiozzo, K.M. Baldwin, Skeletal muscle unweighting: spaceflight and ground-based models, J. Appl. Physiol. 95 (2003) 2185-2201.

[64] E.J. Huang, L.F. Reichardt, Neurotrophins: roles in neuronal development and function, Annu. Rev. Neurosci. 24 (2001) 677-736.

[65] M. Salanova, G. Schiffl, B. Püttmann, B.G. Schoser, D. Blottner, Molecular biomarkers monitoring human skeletal muscle fibres and microvasculature following long-term bed rest with and without countermeasures, J. Anat. 212 (2008) 306-318.

[66] M. Salanova, G. Schiffl, J. Rittweger, D. Felsenberg, D. Blottner, Ryanodine receptor type-1 (RyR1) expression and protein S-nitrosylation pattern in human soleus myofibres following bed rest and exercise countermeasure, Histochem. Cell Biol. 130 (2008) 105-118. 